\title{
CHEMICAL, CLINICAL, AND IMMUNOLOGICAL STUDIES ON THE PRODUCTS OF HUMAN PLASMA FRACTIONATION.
}

\author{
XXI. THE USE OF FIBRIN FOAM AS A HEMOSTATIC \\ AGENT IN NEUROSURGERY: CLINICAL AND \\ PATHOLOGICAL STUDIES 1,2
}

\author{
By ORVILLE T. BAILEY AND FRANC D. INGRAHAM \\ (From the Departments of Pathology and Surgery, Harvard Medical School, and the Surgical \\ Services of the Peter Bent Brigham and The Children's Hospitals, Boston)
}

(Received for publication February 17, 1944)

In neurosurgery, there are several types of bleeding for which the hemostatic agents now in general use are not satisfactory. By far the most common is capillary oozing. The control of such oozing in the dura, brain, spinal cord, and especially the beds of intracranial tumors, frequently prolongs neurosurgical procedures and at times is difficult, if not impossible, to stop completely. Another type of bleeding for which the common hemostatic agents are not satisfactory is that from the dural sinuses and other large venous channels where ligature cannot be applied. The various substances which have been employed in these situations up to the present time have been discussed elsewhere $(1,2)$.

When fibrin foam was prepared by Bering (1) from the products of large scale plasma fractionation $(2,3)$, the investigation of its uses in neurosurgery was undertaken, along with a study of the tissue reactions to its presence. There are now available for evaluation both clinical material and a series of experimental studies in monkeys.

\section{CLINICAL MATERIAL}

Fibrin foam and thrombin have been used for the control of bleeding in 170 neurosurgical patients at the Peter Bent Brigham Hospital and The Children's Hospital, Boston.

\footnotetext{
1 This work has been carried out under contract, recommended by the Committee on Medical Research, between the Office of Scientific Research and Development and Harvard University.

${ }^{2}$ This paper is Number 26 in the series "Studies on Plasma Proteins" from the Harvard Medical School, Boston, Massachusetts, on products developed by the Department of Physical Chemistry from blood collected by the American Red Cross.
}

The thrombin is dissolved in $30 \mathrm{cc}$. of saline solution at room temperature and foam soaked in this solution a few minutes before use. The technic of application to the bleeding point necessarily varies with the circumstances. In general, the area has been dried by suction, the moist foam applied, and a cotton pattie held over the foam until it has become adherent. The foam, applied in this way, has proved to be very effective in controlling oozing from the outer surface of the dura, bleeding from the substance of tumors, and from the tumor bed after removal, as well as from larger vessels, such as large superficial cortical veins and even from dural sinuses.

The material has been left in place in all of these patients in amounts varying from small fragments to a mass the size of a golf ball. In no instance has there been any clinical evidence of cortical irritation. Furthermore, in those patients in whom the operative field was seen after 14 hours to 81 days, either at secondary operation (15 patients) or at autopsy (4 patients), there was no evidence of an inflammatory reaction or other unfavorable result.

The following case history is given to illustrate its use:

A 36-year-old housewife was admitted October 16, 1943 because of failing vision. Two years and four months previously glasses had been fitted but she was not aware of loss of visual acuity until 5 months ago. There had been a mild personality change, of which the patient was not aware, through a 2-year period. Mild headaches had been associated with menses for many years. Examination showed almost total loss of vision in the right eye and ability to count fingers at one foot with the left eye. Roentgenograms revealed erosion of the sella turcica and slight thickening of the right frontal bone. A diagnosis of meningioma was made and a right frontal bone flap was turned down. The tumor involved the frontal bone and 
dura down to and into the right frontal sinus. The involved bone and dura were resected and the large vessels around the tumor clipped or coagulated. A mass filling the entire fossa and displacing the falx to the left of the midline was then mobilized and lifted out. A large ball of foam was immediately placed in the tumor bed which was bleeding freely from numerous points. This was held in place with a piece of lintine (cotton) for several minutes and the central portion of the foam mass then pulled gently out leaving a thin shell of the material lining the bed. At this point there was no bleeding. The cavity was filled with saline, the dural defect replaced with fibrin film (described below) and the flap closed. Recovery was uneventful. Histological diagnosis: Meningioma.

(Note: There is no reason why the entire mass of foam should not have been left in place if necessary. The use of the foam made it possible to remove the entire tumor at one time and to leave the tumor bed entirely free from oozing. This was, moreover, accomplished in a much shorter time than would have been possible without the use of the foam.)

\section{TISSUE REACTIONS OF FIBRIN FOAMS IN HUMAN PATIENTS}

Fibrin foams before implantation. Samples of fibrin foams were fixed in Zenker's fluid, embedded in paraffin, cut, and stained with phloxinemethylene blue. Under these circumstances, the foam was found to consist of a network of dark blue fibers with clear interstices. This is a twodimensional picture; in three dimensions, the structure of the foam would be more like a honeycomb. There was a close similarity in staining reaction between the fibers of the foam and the fibrin of spontaneous blood clots. However, the fibers of the foam were coarser and more homogeneous than were the fibers of freshly clotted blood.

Tissue reactions in human patients. We have

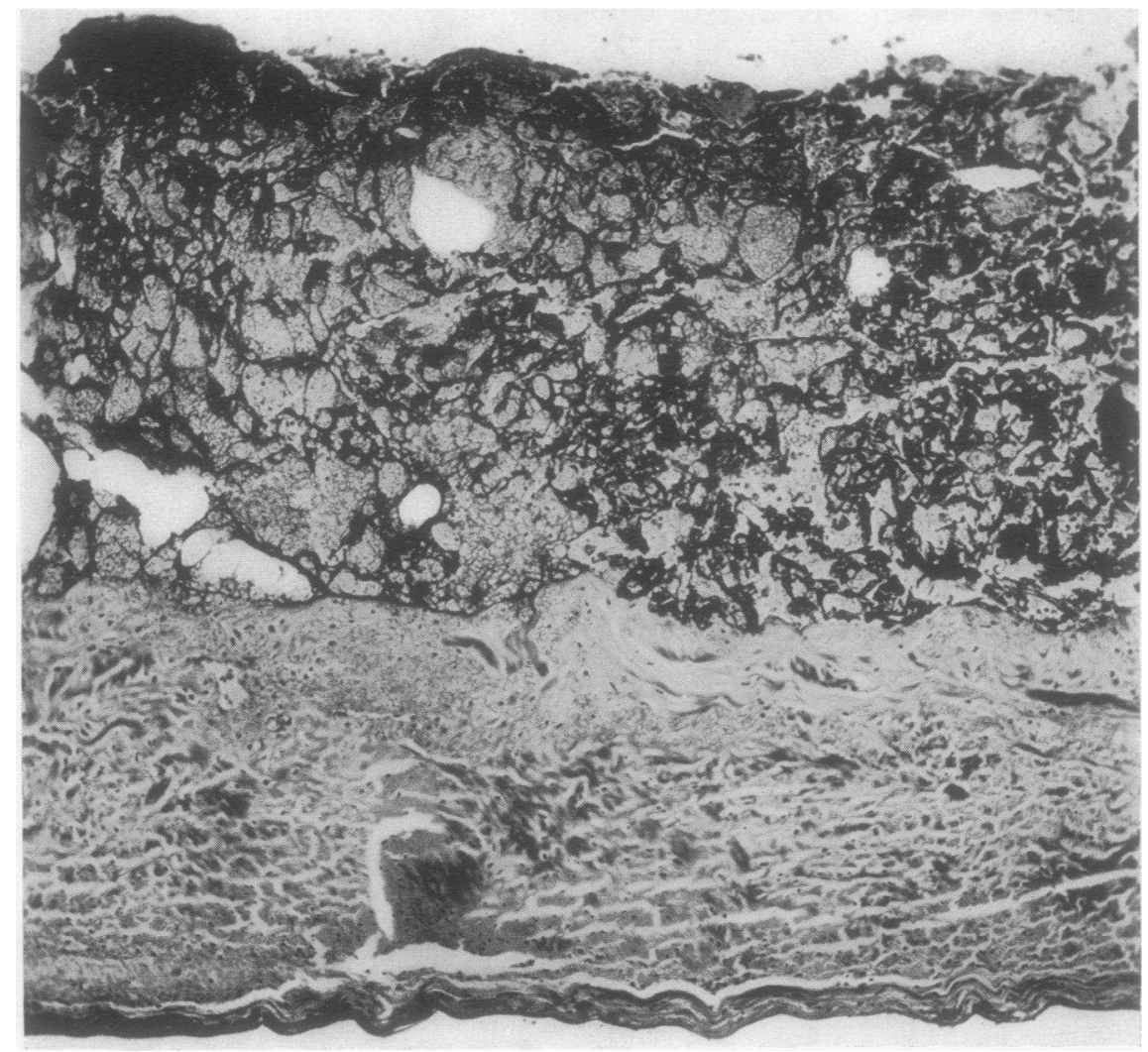

Fig. 1. Low Power Photomicrograph Showing Fibrin Foam on the Superior Surface of The Dura

The specimen was obtained at autopsy, 14 hours after operation. The illustration shows the histological appearance of the foam and the lack of immediate reaction in this patient. 


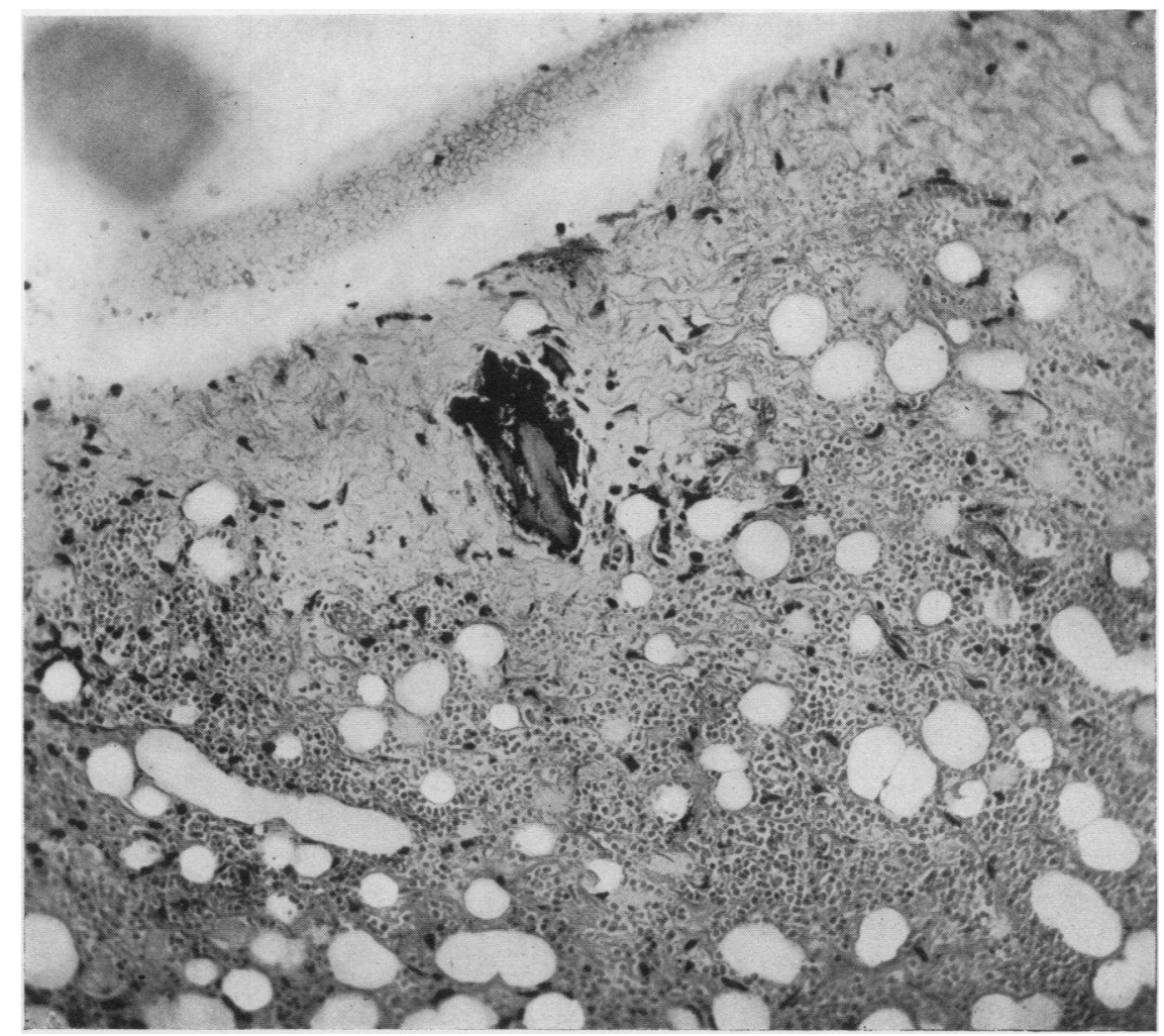

Fig. 2. Photomicrograph Showing a Small fragment of Foam in a Specimen Obtained at Secondary Operation 24 Days After the Original Operation

This was the only fragment of foam identified.

had the opportunity to study the tissue reactions to fibrin foams in 19 human patients. In these patients, foams were removed at secondary operations or (in 4 instances) at autopsy. The length of time that the foam had been in contact with the tissues varied from 14 hours to 81 days. Figure 1 shows the appearance of foam, which had been applied to the dura at operation, 14 hours before death. The appearance of the fibers of the foam was essentially the same as before it had been in contact with the tissues. The interstices, however, had become filled with clotted blood. There was no reaction in the tissues. The complete absence of tissue reaction in this specimen may be due in part to the poor general condition during the 14 hours that the foam was in contact with the dura.

In a specimen of foam obtained 3 days after it had been left on the dura, the total amount of foam was much less than that originally placed there at the time of the operation but consider- able numbers of fibers were easily identified. The foam became eosinophilic at the periphery where it was in contact with living tissues; except for this narrow zone, the foam retained the same appearance as it had before it was placed in the patient. About the foam, there were moderate numbers of polymorphonuclear leukocytes and lymphocytes. Also present were scattered mononuclear cells which contained homogeneous eosinophilic material, judged to be minute fragments of foam.

No large fragments of the foam were found in any of the specimens except those recovered at 14 hours and at 3 days. In specimens obtained from 7 to 24 days after they had been left in place at operation, either a few very small fragments were present or no trace of foam was found.

Figure 2 illustrates the only remnant of the foam which could be identified 24 days after a large amount had been used to control oozing from the dura. There was a small mat of fibrous 
tissue about the fragment and a few mononuclear cells were seen. The material about the fragment was mostly fresh blood from the second operation at which the tissues were removed. Since the foam was used to cause the coagulation of blood, it was inevitable that the foam should at once be surrounded by blood clot. There would be a considerable number of fibroblasts produced in the organization of the blood clot. Hence, it was impossible to determine how many of the fibroblasts were due to the presence of the foam and how many to the blood clot. It appeared from this and other specimens, that the tissue reaction to the foam was less than to a blood clot of comparable size.

Figure 3 shows a portion of cerebral cortex on which fibrin foam had been used as a hemostatic agent 81 days previously. No trace of foam re- mained. While the fibrous tissue of the arachnoid was somewhat increased, this was not in excess of that expected from the operative procedure alone. There were no tissue changes which could be definitely related to the fibrin foam anywhere in the specimen.

\section{ANIMAL EXPERIMENTS}

A series of animal experiments was also carried out to determine the local reaction of cerebral tissues to foam and thrombin. For this study, 37 monkeys (Macaca mulatta) were used to test 6 standard conditions. These not only provided an opportunity to study the tissue reactions to fibrin foam but also included an opportunity to compare the tissue reaction due to foam with that due to soluble cellulose. In each animal,

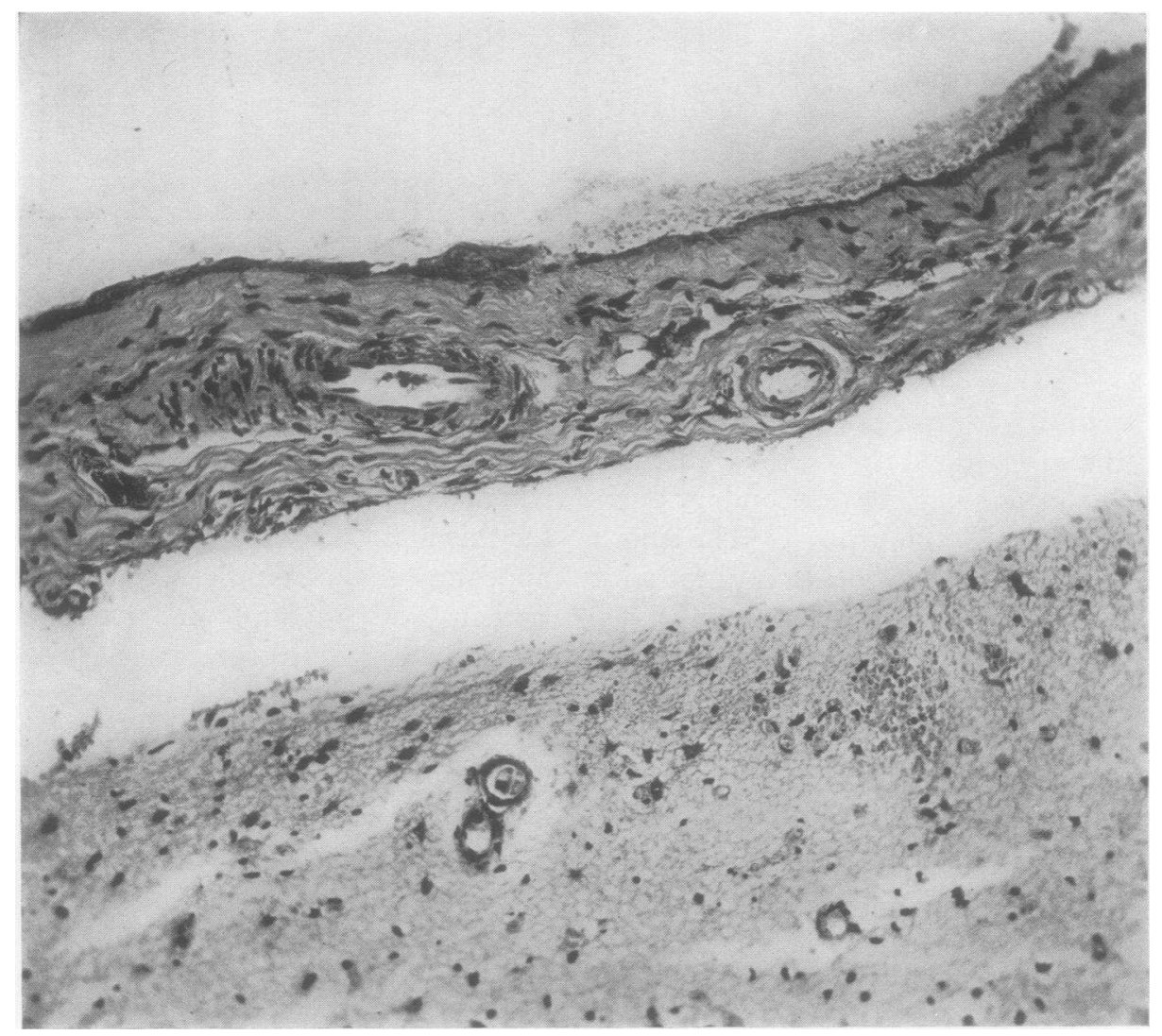

Fig. 3. Photomicrograph of Meninges and Cerebral Cortex Showing the Site at Which Fibrin foam Had Been Placed 81 Days Previously. No Residual foam WAS IDENTIFIED.

While the fibrous tissue of the meninges was increased, the amount was not in excess of that which could be accounted for by the operative procedure alone. 
bilateral craniotomies were performed, the dura opened and the parietal lobe exposed. Foam and thrombin were placed (1) on the normal cortex, (2) on cortex damaged by multiple needle wounds which produced bleeding, and (3) within the substance of the parietal lobe at a depth of 3 to $4 \mathrm{~mm}$. Since it would obviously be desirable to use the material in compound head injuries with gross contamination, particularly under war conditions, the same procedures were carried out using foam and thrombin in combination with sulfadiazine and again with penicillin. The animals were sacrificed at periods varying from 3 hours to 3 months. There was no physiological evidence of cortical irritation or other untoward effects in any of these animals.

When the monkeys were sacrificed, the skulls were fixed in 10 per cent formalin after excess tissue had been trimmed away. The skull was then dissected away so that the relationship of meninges and cerebral cortex could be studied without distortion. Blocks of tissue were then cut, embedded in paraffin, and stained with hematoxylin and eosin.

The earliest tissue reaction in the meninges was the appearance of small numbers of mononuclear cells and polymorphonuclear leukocytes. This was followed by a rapid disappearance of the foam with condensation into a more compact mass. The cellular infiltration, never extensive, became minimal and there was a slight proliferation of fibrous tissue. Only very small bits of foam were present after 1 week and no fragments at all could be identified at 3 weeks.

When relatively large pieces of fibrin foam $(4 \times 4 \times 6 \mathrm{~mm}$.) were inserted directly in the substance of the cerebral cortex, these disappeared at least as rapidly as on the meninges. The maximum tissue reaction noted in any of these monkeys was a slight gliosis at the site of implantation of the foam.

Some of the experiments were planned to provide a basis for comparison of the tissue reactions of fibrin foam and of soluble cellulose soaked in human thrombin solution. All the monkeys in this group had identical operative procedures on each side of the skull as outlined above, but fibrin foam was implanted on one side and soluble cellulose soaked in thrombin on the other. In 2 monkeys, there were adhesions between the arachnoid and dura on the side on which soluble cellulose soaked in thrombin had been placed but no adhesions on the opposite side, where fibrin foam had been implanted. The extent of tissue reaction and rate of absorption were otherwise quite similar with the two hemostatic agents.

When sulfadiazine was placed in experimental wounds containing fibrin foam, there were no detectable changes in the physical characteristics of the foam or in the character or extent of the tissue response. In experimental wounds containing both fibrin foam and penicillin, the properties of the foam were unchanged and the tissue response was not altered. ${ }^{3}$

\section{COMMENT}

From the clinical point of view, fibrin foam has proved to be a valuable hemostatic agent in neurosurgical procedures. It is readily prepared from the dry state in the operating room and the thrombin dissolves quickly. Very little delay, therefore, is occasioned in preparing the material even when vigorous bleeding is encountered unexpectedly.

By the use of the foam and thrombin, as outlined in preceding paragraphs, complete control of oozing is secured. This applies not only to bleeding in such situations as the dura where the more conventional methods are adequate but time consuming, but also to such locations as the beds of neoplasms, where the conventional agents are not always satisfactory. It is also of great assistance in controlling more vigorous bleeding from large venous channels, such as the dural sinuses and cerebral veins. Fibrin foam, however, is seldom effective in the management of bleeding from large arteries.

The material has a conspicuous advantage over muscle in that it could be made easily available in any desired quantity and in that it causes less tissue reaction than does muscle.

In this series of cases, the foam has frequently saved considerable time and has made it possible to accomplish complete extirpation of a tumor which might otherwise have been impossible or

\footnotetext{
${ }^{3}$ The penicillin was provided by the Office of Scientific Research and Development from supplies assigned by the Committee on Medical Research for clinical investigations recommended by the Committee on Chemotherapeutic and Other Agents of the National Research Council.
} 
would have had to be divided into two or more stages.

The tissue reactions to the foam are minimal but not entirely absent. Not only are they slighter than the reaction caused by human muscle when used as a hemostatic agent, but they are also considerably less than those caused by silk and catgut sutures or bone wax. The amount of fibrous tissue produced is more nearly comparable to that resulting from the organization of a small blood clot. As a matter of fact, it is difficult to determine whether the small amount of fibrous tissue left at the site of implantation of foam is due to the foam itself or to organization of the blood clot which the foam has induced. It is possible that these minimal tissue reactions may be further reduced by changes in the methods of preparation of the foams so that they are absorbed even more quickly.

The experimental evidence indicates that the foam may be used in wounds treated with sulfadiazine and penicillin without change in tissue reaction. Clinical experience in a few instances is in accord with the experimental studies on this point.

Additional illustration and discussion of the utility of fibrin foam has been presented elsewhere $(1,4)$.

\section{SUMMARY}

Fibrin foam has been tested under a variety of experimental conditions in a series of monkeys.
The tissue reactions were minimal in all animals, including those in which the foam was implanted in conjunction with sulfadiazine and penicillin.

The foam was used in 170 neurosurgical patients, under varying conditions. Its rapid hemostatic action has shortened many operations. In some instances, it has made successful operation possible when other hemostatic agents would have been inadequate.

\section{BIBLIOGRAPHY}

1. Bering, E. A., Jr., Chemical, clinical, and immunological studies on the products of human plasma fractionation. XX. The development of fibrin foam as a hemostatic agent and for use in conjunction with human thrombin. J. Clin. Invest., 1944, 23, 586.

2. Edsall, J. T., Ferry, R. M., and Armstrong, S. H., Jr., Chemical, clinical, and immunological studies on the products of human plasma fractionation. XV. The proteins concerned in the blood coagulation mechanism. J. Clin. Invest., 1944, 23, 557.

3. Cohn, E. J., Oncley, J. L., Strong, L. E., Hughes, W. L., Jr., and Armstrong, S. H., Jr., Chemical, clinical, and immunological studies on the products of human plasma fractionation. I. The characterization of the protein fractions of human plasma. J. Clin. Invest., 1944, 23, 417.

4. Ingraham, F. D., and Bailey, O. T., The use of products prepared from human fibrinogen and thrombin in neurosurgery. Fibrin foams as hemostatic agents; fibrin films in repair of dural defects and in prevention of meningocerebral adhesions. J. Neurosurg., 1944, 1, 23. 